\title{
LES BESOINS DES ORGANISMES D'ÉDUCATION DES ADULTES
}

\section{ÉTUDE / RAPPORT DE RECHERCHE}

Aleksander Kobylarek, Luba Jakubowska, Kamil Błaszczyński, Noémie Govindin, Piera Sciama, Alcidio Jesus, Claudia Amaral, Samuel Yosef, Amelia Fiorillo 


\section{LES BESOINS DES ORGANISMES}

D'ÉDUCATION DES ADULTES

ÉTUDE / RAPPORT DE RECHERCHE

Aleksander Kobylarek, Luba Jakubowska, Kamil Błaszczyński, Noémie Govindin, Piera Sciama, Alcidio Jesus, Claudia Amaral, Samuel Yosef, Amelia Fiorillo 
The publication prepared as a result of The Erasmus + Strategic Partnership Project in the Field of Adult Education titled Needs of adults education stakeholder ( $\mathrm{N}^{\circ}$ 2019-1-PLo1-KA204-065792) with experts from the consortium organizations: FUNDACIA PRO SCIENTI PUBLICA (Poland), ITAKA TRAIIING (Italy), E-SENIORS: INTIATION DES SENIORS AUX NTIC ASSOCLATION (France) and AFN ACADEMIA FORMAÇ̣̄o DO NORTE (Portugal).

The European Commission's support for the production of this publication does not constitute an endorsement of the contents, which reflect the views only of the authors, and the Commission cannot be held responsible for any use which may be made of the information contained therein.

AUTHORS:

Aleksander Kobylarek, Luba Jakubowska, Kamil Błaszczyński, Noémie Govindin, Piera Sciama, Alcidio Jesus, Claudia Amaral, Samuel Yosef, Amelia Fiorillo

STATISTICAL EDITORS:

Kamil Blaszzzyński, University of Wroclaw, Poland

REVIEWER -

Tijana Milenkovic Jankovic, The uK

TANSLATON INTO FENCH

Noémie Govindin, Piera Sciama, France

DESIGN AND DTP

Hanna Włoch, Polan

Foundation Pro Scientia Publica

Ul. Redycka 37, 51-169 Wroclaw

www.proscientiapublica.pl
Le soutien de la Commission européenne à la production de cette publication ne constitue pas une approbation du contenu, qui reflète uniquement le point de vue des auteurs, et la Commission ne peut pas être tenue responsable de toute utilisation qui pourrait être fite des informations qu'elle contient.

\section{Février 2021}

Creative Commons Attribution 4.0 International

Authority code: CC_BY_4_0

(c) (i)

BNN 978-83-953451-3-5

doi: 10.15503/andr.2021.neon.fr

Photo by

CoWomen ; Green Chameleon ; krakenimages

Marten Bjork ; Sarah Dorweiler ; You X Ventu

on Unsplash

$$
\begin{aligned}
& \text { Introduction } 4 \\
& \text { Résultats } 10 \\
& \text { Résumé } 24 \\
& \text { References } 30 \\
& \text { Annexes } 34 \\
& \text { Annex } 36 \\
& \text { Annex } 11 \quad 40
\end{aligned}
$$


1. Introduction

\section{INTRODUCTION}


Le projet « Besoins des organismes d'éducation des adultes» $\left(\mathrm{N}^{\circ}\right.$ 2019-1-PLo1- KA204065792) a été créé en réponse à l'absence d'un outil permettant de mesurer les besoin du personnel du secteur de l'éducation des adultes.

Malgré le développement soutenu de la méthodologie de l'andragogie, il existe toujours un manque d'outils de recherche spécifiques à ce domaine. L'andragogie utilise des outils provenant de domaines connexes, principalement de la psychologie. Une telle solution n'est pas satisfaisante, d'une part parce que de nombreux outils sont pe accessibles (principalement en raison de la nécessité de payer des frais d'utilisation et de la nécessité de la présence d'un psychologue dans l'équipe) et, d'autre part, parce qu'ils ne sont pas spécifiques à l'étude des besoin des organisations du secteur de l'éducation des adultes et des besoins éducatifs de leurs employés.

En réponse au constat susmentionné, les responsables du projet ont élaboré un questionnaire pour évaluer ces besoins. L'enquête a été traduite dans quatre langues. Sur quête a été traduite dans quatre langues. Su recueillies, une classification des besoin éducatifs du personnel du secteur de l'éducation des adultes a été créée (Aleksande Kobylarek, Luba Jakubowska, Noémie Govindin, Piera Sciama, Alcidio Jesus, Claudia Amaral, Samuel Yosuf, Amelia Fiorillo).

Ce rapport contient des informations su les besoins en matière d'éducation et l'évaluation quantitative des organisations d'éducateurs d'adultes et des travailleurs de ONG dans les pays du consortium (Pologne, France, Italie et Portugal), dans d'autre pays de l'UE et certains pays tiers. L'enquête a réuni 1176 participants. Outre nombre représentatif de répondants, il est important de souligne a été menée dans différents pays, et non pas uniquement dans les pays du consorium, garantissant que les résultats obtenus sont révélateurs des besoins du secteur de l'éducation des adultes en Europe.

OUTILS DE DIAGNOSTIC

DES COMPÉTENCES CLÉS ET LES BESOINS DE CHANGEMENT DANS L'ORGANISATION

Afin de diagnostiquer les besoins en matière d'éducation, un questionnaire rédigé par lauteur, appelé outil d'inventaire des besoins en éducation des adultes (AENI) a été utilisé Le questionnaire est un outil conçu pour étudier les compétences clés des travailleurs de léducation des adultes (Conseil de l'Unio . Le modèle de compétence s'inscrit dans le concept de l'apprentissage tout au long de la vie et ne se concentre pas sur la fonction de compensation du processus éducatif; au lieu de cela, il favorise la perspicacité de l'éducateur dans son développement personnel et sa participation consciente et active. L'AENI a été construite sur la base de la recommandation de l'Union uropéenn conces clés u 21e siècle. Sur la base de ces recommandations, l'équipe de recherche a créé, testé et évalué l'AENI à l'aide de méthodes lyse statistique.

La fiabilité du test a été confirmée par intercorrélation. La fiabilité du questionnaire a été confirmée par l'analyse factorielle. De plus, la fiabilité théorique a été inspectée à travers la corrélation de l'AENI avec le Socia Competencies Profile (РRокоS) (Martowska, Matczak, 2013), qui mesure les compétences sociales dans les domaines suivants: assertivité, coopération, esprit social, débrouillardise et conscience communautaire. Afin détudier la fiabilité théorique, des hypothèses ont été formulées à la fois sur la corrélation des scores généraux (issus des ques- tionnaires AENI et PROKOS) et sur les échelles sélectionnées. Des études de corrélation, auxquelles ont participé 98 répondants, on confirmé la fiabilité théorique du questionnaire AENI.

Avant d'implémenter la recherche, une analyse différenciée a été menée sous la forme d'un test de fiabilité Alpha-Cronbach (avec 210 participants) qui a révélé une très grande fiabilité de l'outil créé $(\alpha=0.846$ de Cronbach) et de ses dimensions.

Le questionnaire développé (AENI) fourn deux informations majeures : le score général d'un individu et les scores dans six souscatégories de diagnostic :

1) compétences en communication ( $\mathrm{CC}$ ) 2) compétences multilingues et multiculturelles (ML\&MC)
3) compétences numériques (DC) ; 4) compétences entrepreneuriales (EC) ; 5) ouverture à la science et à la culture (OSC) ; 6) compétences sociales et civiques (SCC).

Toutes les sous-catégories ont été créées par l'équipe de recherche polonaise (Kobylarek, Jakubowska, Błaszczyński 2020 - non publié officiellement à ce stade), en utilisant des outils statistiques tels que 1) l'analyse des facteurs principaux, 2) le test de fiabilité alpha de Cronbach et 3) le test d'association de Kendall W.

Chaque individu peut obtenir un maximum de $100 \%$ des résultats du test général et des sous-catégories. Plus le score est proche de $100 \%$, moins les besoins éducatifs de l'individu sont visibles.

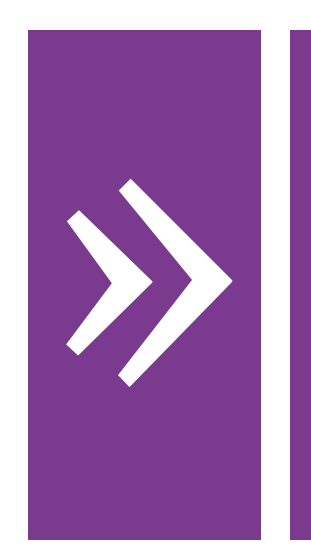

Pour ce rapport, la classification des scores utilisée est la suivante :

20-44 \% - faible score - besoins éducatifs fortement ressentis

45-75 \% - moyen - besoins ressentis en matière d'éducation ressentis de manière modérée

76-10o \% - élevé - besoins en matière d'éducation faiblement ressentis

Afin de donner aux répondants l'opportunité d'évaluer leur organisation, uneenquête en 18 points a été menée. L'enquête était composée de 18 énoncés représentant 18 catégories (tableau 1) pour lesquelles les répondants étaient invités à évaluer leur attitude respective sur une échelle de 5 points allant de « pas du tout d'accord » à « tout à fait d'accord ». Les éléments inclus dans l'enquête ont été créés sur la base de la théorie de deux facteurs de Herzberg sur la motivation pour le travail (source : Bassett-Jones, Lloyd, 2005).

Les scores ont été mesurés en pourcentage. Plus le score obtenu par l'individu était élevé, meilleure était son opinion sur son organisation et moins il est nécessaire de changer lorganisation. 
Tableau 1. Éléments d'évaluation de l'organisation - Liste et description

\begin{tabular}{|c|c|}
\hline Appréciation de l'employeur & L'employé(e) se sent apprécié(e) par son employeur. \\
\hline Appréciation du chef & $\begin{array}{l}\text { L'employé(e) se sent apprécié(e) par son/sa chef(fe) } \\
\text { direct(e) ou son superviseur. }\end{array}$ \\
\hline $\begin{array}{l}\text { Atteinte des objectifs de l'or- } \\
\text { ganisation }\end{array}$ & $\begin{array}{l}\text { L'employé(e) peut atteindre les objectifs fixés par son } \\
\text { employeur. }\end{array}$ \\
\hline Réussite personnelle & $\begin{array}{l}\text { L'employé(e) ressent un succès personnel au sein de l'or- } \\
\text { ganisation. }\end{array}$ \\
\hline Responsabilité & $\begin{array}{l}\text { L'employé(e) se sent fortement responsable des tâches } \\
\text { qu'il/elle accomplit. }\end{array}$ \\
\hline Indépendance & $\begin{array}{l}\text { L'employé(e) dispose d'une grande liberté dans l'accom- } \\
\text { plissement de la tâche qui lui a été confiée. }\end{array}$ \\
\hline Progrès & $\begin{array}{l}\text { L'employeur offre à l'employé(e) des possibilités de pro- } \\
\text { gression. }\end{array}$ \\
\hline Développement personnel & $\begin{array}{l}\text { Le travail actuel a un impact positif sur le développement } \\
\text { personnel de l'employé(e). }\end{array}$ \\
\hline $\begin{array}{l}\text { Stratégie de développement de } \\
\text { l'organisation. }\end{array}$ & $\begin{array}{l}\text { L'organisation de l'employé(e) a une stratégie de dévelop- } \\
\text { pement claire et l'employé(e) la comprend. }\end{array}$ \\
\hline $\begin{array}{l}\text { Continuité du développement } \\
\text { de l'organisation. }\end{array}$ & $\begin{array}{l}\text { L'organisation ne met pas fin aux tentatives d'amélioration } \\
\text { et s'efforce continuellement de perfectionner et d'étendre } \\
\text { son activité. }\end{array}$ \\
\hline Lieu de travail & $\begin{array}{l}\text { L'organisation fournit à l'employé(e) un lieu de travail } \\
\text { adéquat pour accomplir les tâches qui lui sont confiées. }\end{array}$ \\
\hline Confort & L'organisation met l'accent sur le confort des employé(e)s. \\
\hline Salaire & L'employé(e) est satisfait(e) de son salaire. \\
\hline Avantages et primes & $\begin{array}{l}\text { L'organisation offre à l'employé(e) une vaste gamme } \\
\text { d'avantages et de primes supplémentaires. }\end{array}$ \\
\hline Relations avec l'employeur & $\begin{array}{l}\text { L'employé(e) entretient de bonnes relations personnelles } \\
\text { avec son employeur. }\end{array}$ \\
\hline Relations avec les collègues & $\begin{array}{l}\text { L'employé(e) entretient de bonnes relations personnelles } \\
\text { avec ses collègues de travail. }\end{array}$ \\
\hline Sécurité de l’emploi & $\begin{array}{l}\text { L'organisation est financièrement stable et donne à l'em- } \\
\text { ployé(e) un sentiment de stabilité de l'emploi. }\end{array}$ \\
\hline Travail et vie privée & $\begin{array}{l}\text { Les tâches effectuées dans l'organisation ont un effet néga- } \\
\text { tif sur la vie privée de l'employé(e). }\end{array}$ \\
\hline
\end{tabular}

(source: Auteurs propres sur la base de: Herzberg, Mausner, Snyderman, 1959)
INTERPRÉTATION DES RÉSULTATS DE L'ÉTUDE SUR LA NÉCESSITÉ DE CHANGER LES ORGANISATIONS

Les scores ont été mesurés en pourcentage. Chaque élément pouvait obtenir un score minimum de o \% et un score maximum de $100 \%$. Plus le score obtenu par l'individu était élevé, meilleure était son opinion sur son organisation et moins il est nécessaire de changer l'organisation. La seule exception était la dernière catégorie axée sur le travail et la vie privée. Dans cette catégorie, plus le score obtenu était faible, plus l'influence du travail sur la vie privée était faible, donc plus le score était élevé.

Il est recommandé d'interpréter les scores obtenus lors de l'évaluation de l'organisation selon la classification proposée ci-dessous :

0-20 \% - très faible - très mauvaise opinion de lorganisation/employeur

21-40\% - faible - mauvaise opinion de l'organisation/employeur

41-6o\% - moyen - opinion neutre sur l'organisation/l'employeur

61-80\% - élevé - bonne opinion de l’organisation/employeur

81-100\% - très élevé - très bonne opinion sur l'organisation/employeur

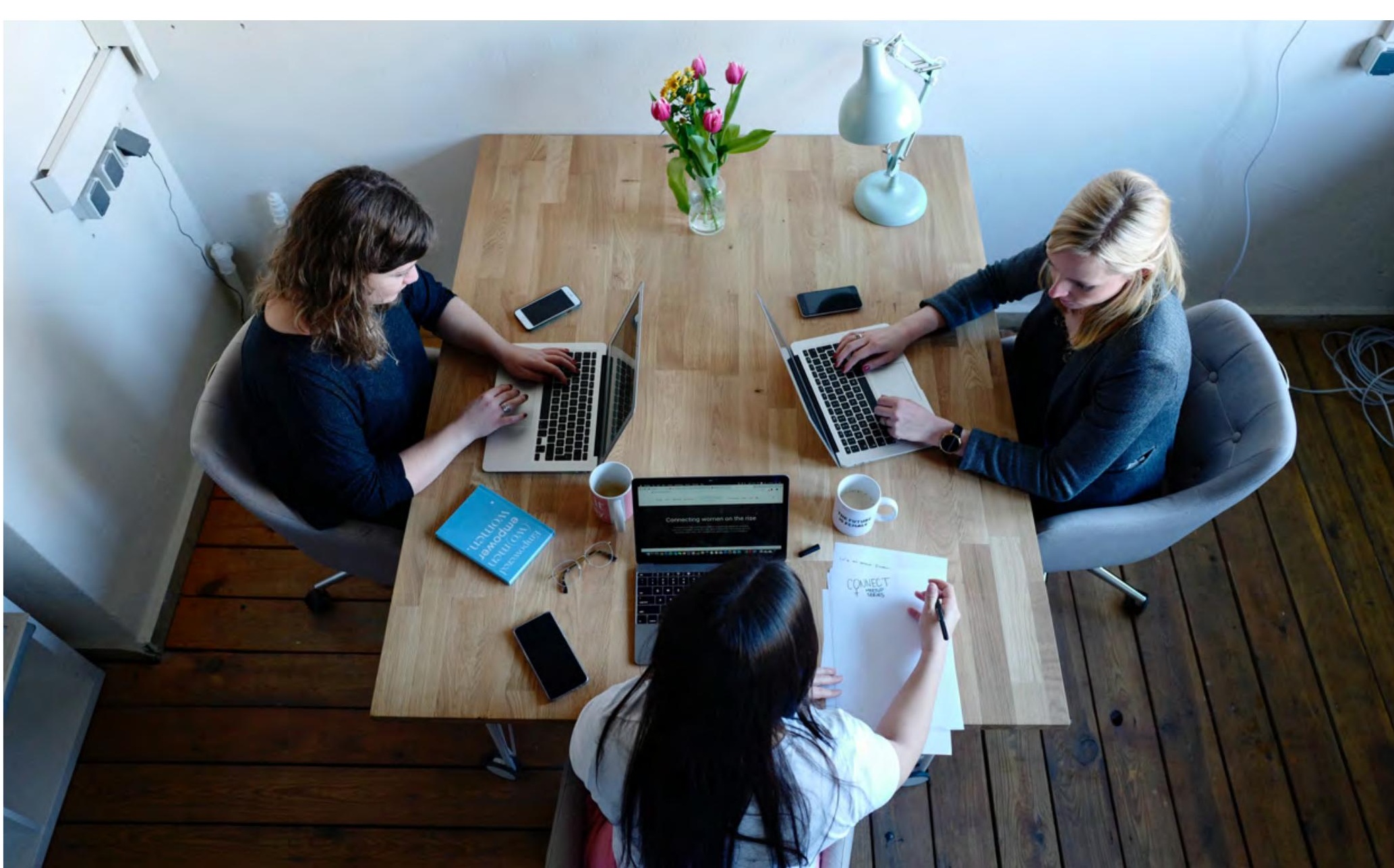


2. Résultats

\section{RÉSULTATS}


RÉSULTATS GÉNÉRAUX POUR LES COMPÉTENCES RESPECTIVES DANS LE TEST AENI

Les résultats du diagnostic réalisé ont été divisés en trois niveaux d'analyse: 1) scores généraux obtenus par tous les participants de l'étude ; 2) comparaison des scores obtenus par les participants qui étaient des citoyens d'un pays de l'ue par rapport aux participants des pays non-UE ; 3 ) comparaison des scores obtenus par les participants des pays du consortium : France, Italie,
Pologne et Portugal. 1176 participants ont pris part à létude, le score général moyen du test AENI était de $78 \%$, ce qui peut être classé comme bon. L'analyse détaillée des sous-catégories de l'AENI a révé que le score le plus bas a été obtenu dan les compétences entrepreneuriales (73\%) qui peuvent être classées comme bonnes. Et les scores les plus élevés ont été obtenus dans les compétences numériques (82\%) et les compétences sociales et civicomme très bonnes.
Image 1. Test pour un inventaire des besoins dans l'éducation des adultes Notes moyennes des sous-catégories

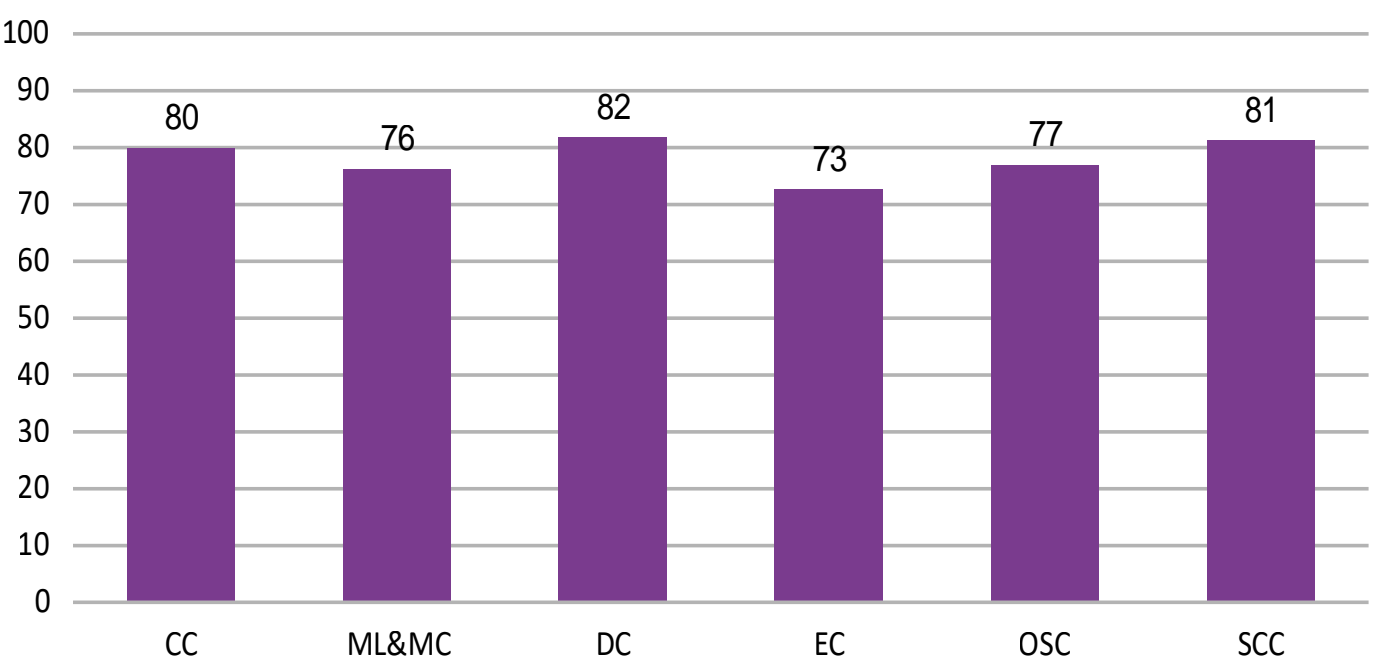

DIAGNOSTIC DES BESOINS DE CHANGEMENT DANS LES ORGANISATIONS

Les résultats obtenus lors de l'enquête d'évaluation des organisations ont révélé un scor général moyen de $69 \%$, qui peut être considéré comme élevé. L’analyse des catégories individuelles a révélé que le score le vail et la vie privée, bien qu'en raison de l'échelle inver- sée de la catégorie, nous pouvons supposer que la plupart des participants ne tiennent pas compte des effets négatifs de leur travail sur la vie privée, ce qui est un phénomène positif. Parmi les autres scores faibles notables, on peut citer les salaires (51 \%) et les bénéfices/ primes (42\%) Par conséquent, les éducateurs d'adultes et les travailleurs des ONG jugent les avantages matriels quils retere lui-même comme étant médiocres. ages novetiels qu'ils retirent du traval
Image 2. Évaluation de l'enquête sur les organisations

Notes moyennes des sous-catégories

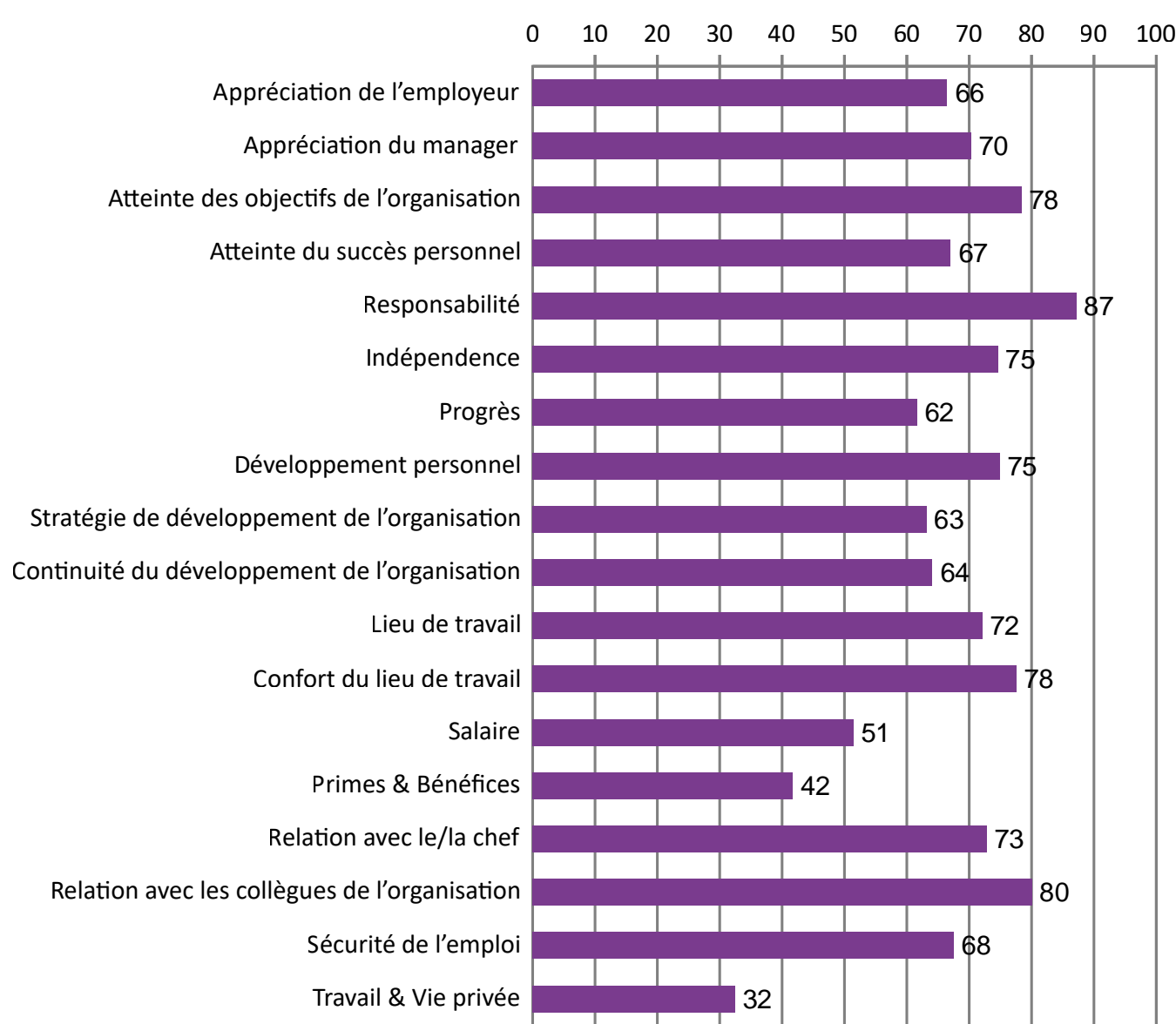

LA COMPARAISON DES NIVEAUX DE COMPÉTENCES CLÉS ENTRE LES RÉPONDANTS DE L'UNION EUROPÉENNE ET DES NON-UE

Dans l'étude, on a enregistré 1049 répondants de l'UE et 107 répondants des non-UE pays d'où que la disparité entre les deux groupes soit visible.

Le score moyen général du test AENI était de $78 \%$ pour les participants de l'ue et de $75 \%$ pour les participants non-UE. Les résultats obtenus montrent donc qu'il y a eu une légère différenciation des cateurs d'adultes travaillant dans le secteur de l'éducation des adultes de l'ue ne sont plus satisfaits que leurs homologues des pays non-UE. L'analyse plus détaillée axée sur les sous-catégories du test AENI a révélé une différenciation des scores dans les catégories communication, numérique, entrepreneurariat et ouverture à la science. Dans toutes ces catégories, les participants de l'UE ont obtenu des scores plus élevés. La plus grande différenciation des scores, de l'ordre de $8 \%$, a été notée dans la catégorie ouverture à la science et à la culture. On peut donc supposer que les répondants de l'ue sont plus satisfaits de leurs compétences en communication, en numérique et surtout en ouverture à la science et à la culture que leurs homologues des pays non-UE. 
Image 3. Résultats des sous-catégories de tests de l'AENI

UE - non-UE. Comparaison des participants

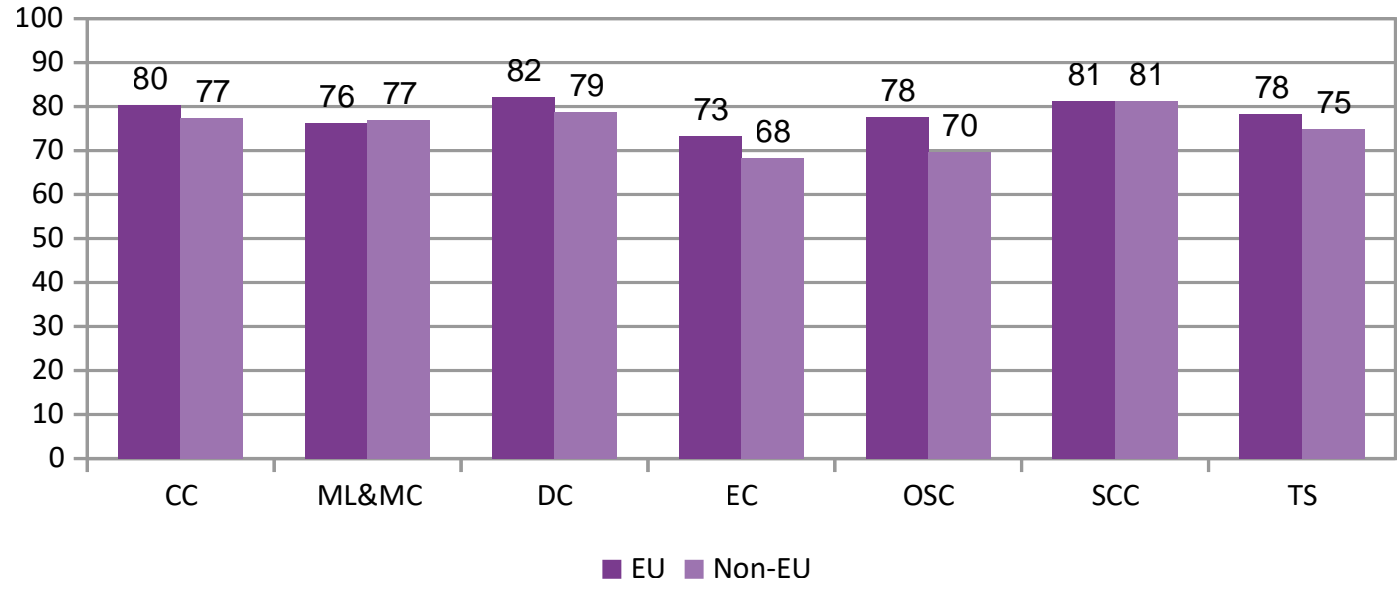

LA COMPILATION DES BESOINS

EN MATIÈRE D'ÉVALUATION

DU CHANGEMENT DANS LES

ORGANISATIONS DE L'UE ET NON-UE

Lévaluation des résultats de l'organisation obtenus par l'enquête a révélé que les notes moyennes générales de nos groupes comparés de répondants étaient de $69 \%$ pou les participants de l'Ue et de $67 \%$ pour les participants hors UE. Ainsi, les participants de l'UE évaluaient leurs organisations de manière légèrement plus positive que leurs non-UE. Une analyse plus détaillée (image 4) des catégories individuelles a révélé une différenciation plus ou moins visible des scores dans toutes les catégories analysées. Les différences les plus visibles ont été relevées dans les domaines de la réussite personnelle (4\%), de la responsabilité (6\%) de l'indépendance ( $6 \%)$, de l'avancement (5\%), du salaire (5\%), des avantages et des primes $(5 \%)$, des relations avec l'employeur $(4 \%)$, de la sécurité de l'emploi (6\%) et de la vie professionnelle et privée ( $8 \%$ ). de réussite personnelle, créent plus de possitages et des primes plus attrayants.
Image 4. Évaluation de l'organisation

Notes moyennes dans les catégories. Comparaison UE \& hors UE

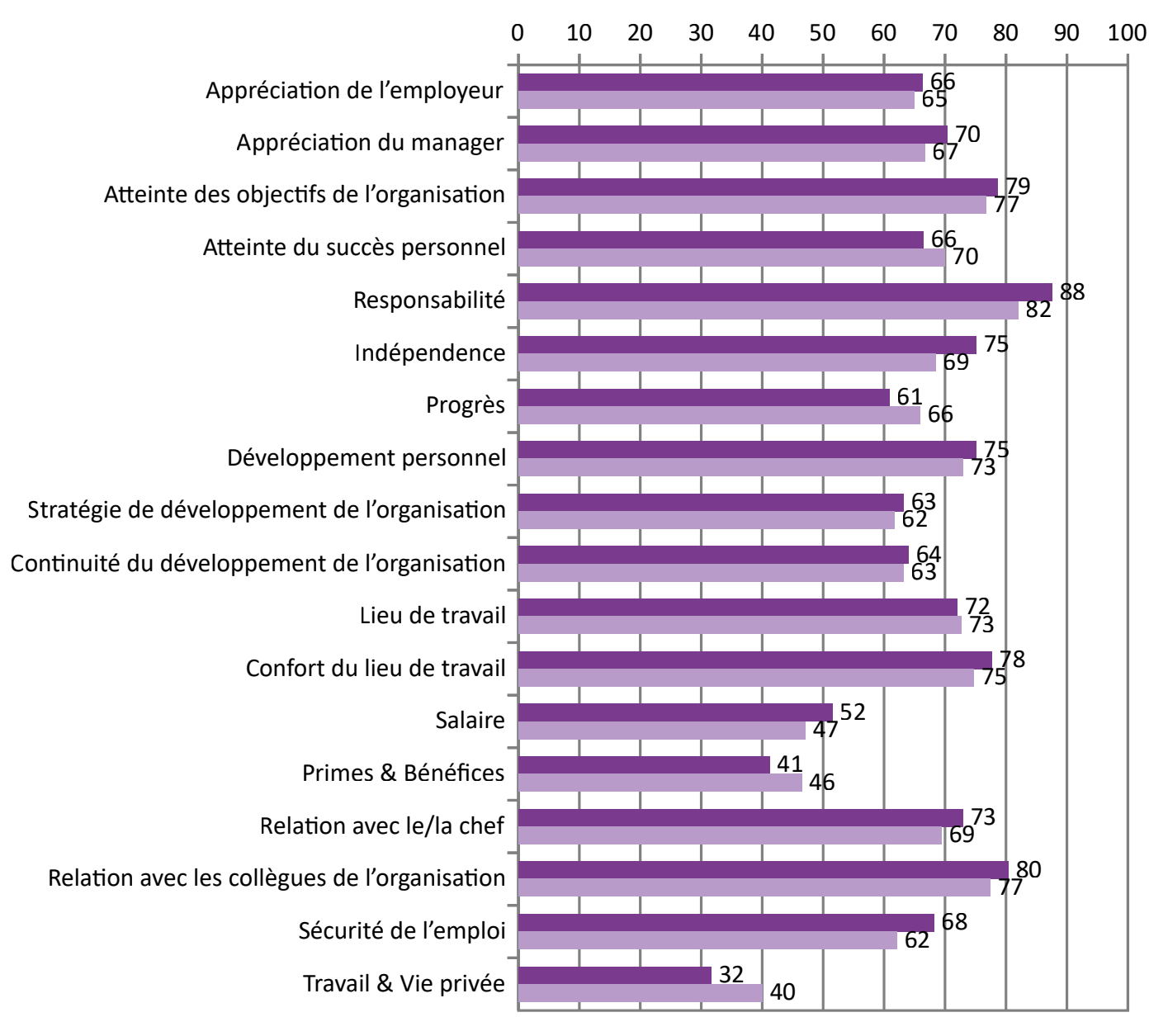

$\square$ Non-EU $\square$ EU
COMPARAISON DES NOTES

MOYENNES GÉNÉRALES DES PAYS

DU CONSORTIUM AU TEST DES

COMPÉTENCES CLÉS DE L'AEN

L'étude a enregistré 201 participants du Portugal, 195 participants d'Italie, 135 participants de France et 159 participants de Pologne. La comparaison des résultat moyens constatés dans les différents pays a révélé (image 5) que le score le plus élevé a été enregistré en Italie (79\%), que des résultats très similaires mais plus faibles ont été constatés en France et au Portugal ( $78 \%$ ) et que le plus faible a été enregistré en Pologne (76\%). Toutes les différences relevées peuvent être classées comme très élevées. donné à leurs employés plus de possibilités bilités d'avancement et proposent des avan- 
Image 5. Test AENI

Comparaison des scores généraux des pays

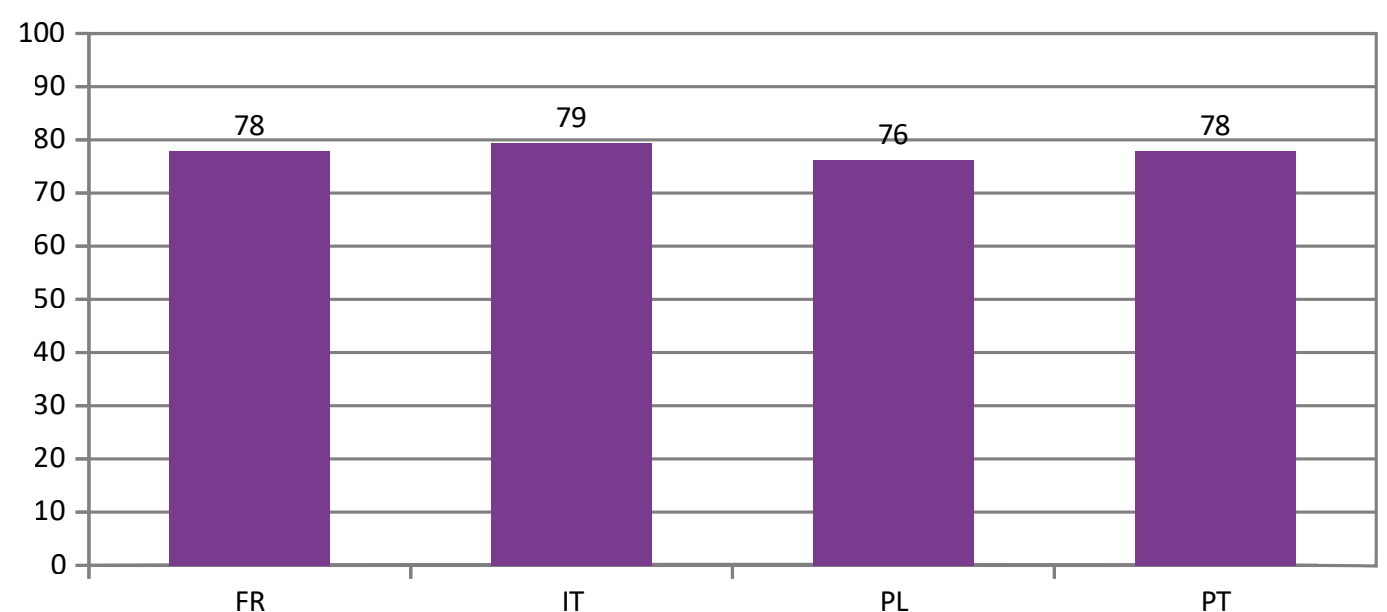

COMPARAISON DES SCORES MOYENS DES PAYS DES PARTENAIRES DU CONSORTIUM AU TEST AENI POUR

LES COMPÉTENCES CLÉS RESPECTIVES

La comparaison des résultats dans les catégories respectives et leur analyse a révélé que la plus grande diversité de scores était notée dans les domaines multilingue et multiculturel et entrepreneuriat. Dans le domaine multilingue et multiculturel, le score le plus élevé a été noté par les participant italiens ( $77 \%$ ) et le plus bas par les participants polonais (69\%). Des scores moyens ont été notés en France et au Portugal mais ils étaient plus proches des scores obtenus par l'échantillon italien. Sur la base des résultats obtenus, on peut supposer que l'échant er que lech hallon polon pe distingue négativenent des groupes comparés. Ainsi, les éducateurs d'adultes et les travailleurs des ONG polonaises peuvent avoir des

besoins éducatifs plus soutenus en ce qui concerne les questions multilingues et multiculturelles.

La deuxième diversité la plus visible dans les résultats a été notée dans le domaine de les résultats a etén notée dans le domaine de l'entrepreunariat, où le score le plus élevé été obtenu par les italiens ( $76 \%$ ). Le score le plus bas a été une fois de plus relevé en Pologne (70 \%). Un score plus bas par rapport à l'échantillon italien a également été noté par l'échantillon français $(72 \%)$. Bien que les scores des echais et français puissent être classés comme bons, ils montrent clairement que certain domaines de l'éducation en matière de compétences entrepreneuriales doivent être améliorés par les répondants. Un score in

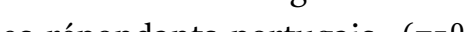
les répondants portugais $(75 \%)$ dans la sous-catégorie « ouverture à la science et à la culture ».
Image 6. Sous catégories du test AENI

Comparaison des scores moyens des pays
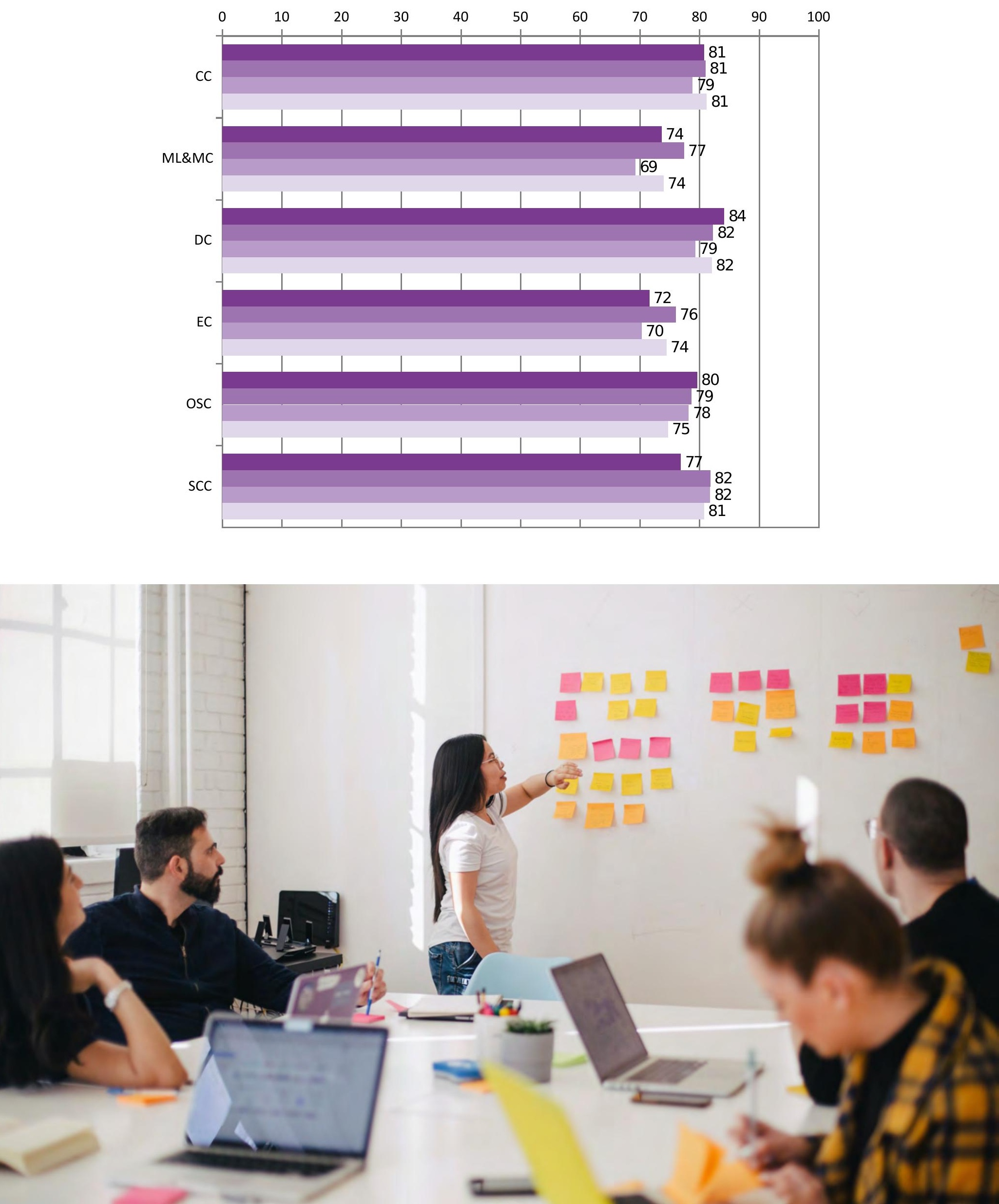
ÉVALUATION DES BESOINS

DE CHANGEMENT DANS LES

ORGANISATIONS

Les résultats de l'enquête sur l'évaluation des organisations ont révélé que le score

Image 7. Évaluation de l'organisation

score moyen général. Comparaison entre les pays

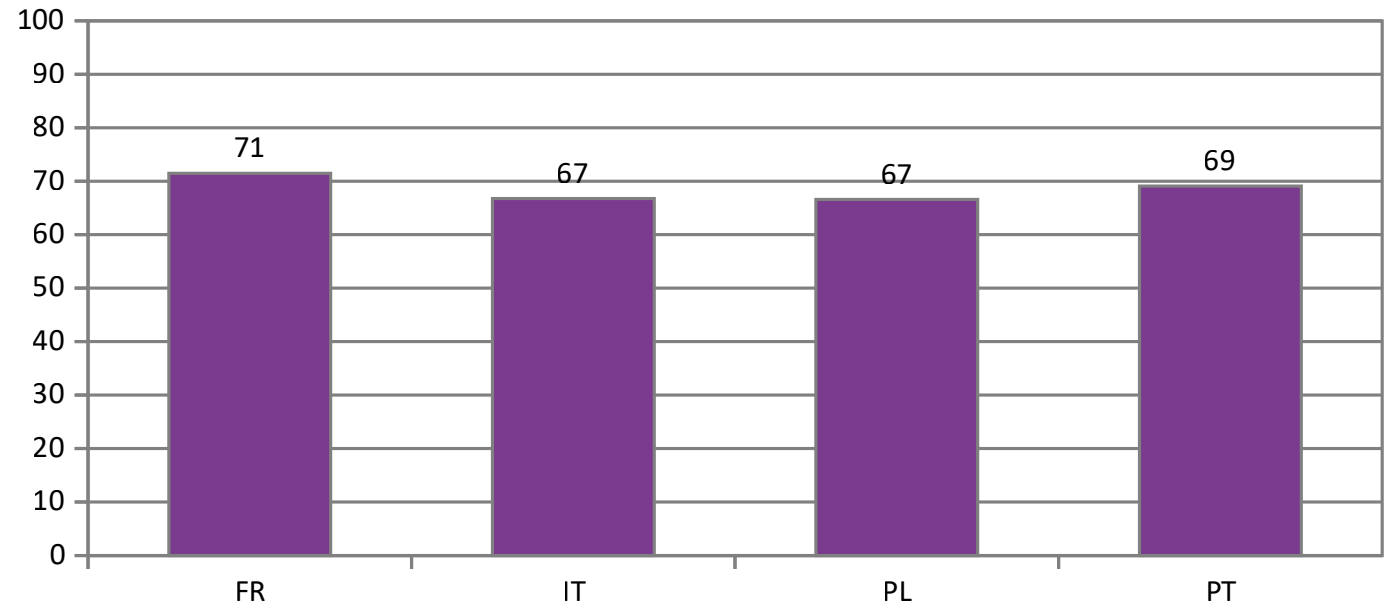

moyen le plus élevé dans l'évaluation des organisations était relevé en France (71 \%), légèrement supérieur qu' au Portugal (69 \%) qu' en Italie et en Pologne (67\% $\%$ ). Toutes les notes peuvent être classées comme bonnes. perçue de la même façon en France, en Italie et au Portugal, à l'exception notable de la Pologne (65\%). L’impact néga-

Image 8. Évaluation de l'organisation Comparaison des scores moyens des pays

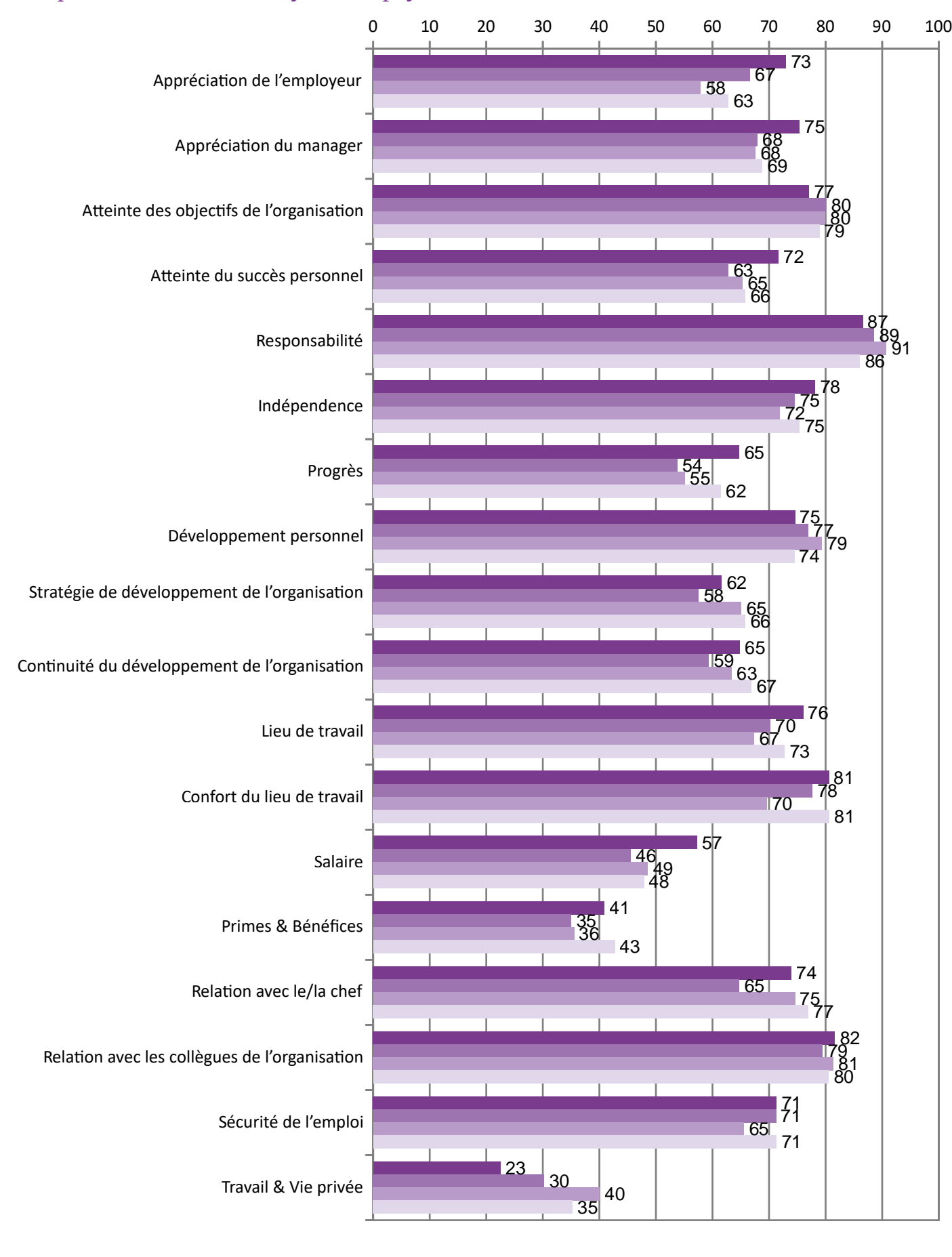

PT $\sqcap$ PL $\sqcap$ IT $\sqcap$ FR tif du travail sur la vie personnelle est le plus important en Pologne (40 \%), le plus faible en France (23\%).
Une fois divisés en catégories (image 8), les résultats ont révélé une très grande variété de scores dans de nombreux domaines. La plus grande appréciation de l'employ eur a été notée en France (73\%) et la plus faible en Pologne $(58 \%)$. L'appréciation $\mathrm{du} /$ de la supérieur hiérarchique est visiblement plus élevée en France (75 \%) que dans tout autre pays. La réalisation des objectifs de l'organisation est la plus élevée en Italie et en Pologne ( 80 \%). C'est en France que la réussite personnelle est la plus élevée (72\%). Le plus grand sentiment de responsabilité a été noté dans l'échantillon polonais (91\%). Le niveau d'indépendance est le plus élevé dans l'échantillon français (78\%). Le niveau de progression le plus élevé est relevé en France (65\%). Les plus grandes possibilités de développement personne ont été relevées dans léchantillon polonais
(79 \%). La stratégie de développement de l'organisation est également la plus élevée dans les échantillons polonais ( $65 \%$ ) et portugais (66\%). La continuité du développement est la plus élevée au Portugal $(67 \%)$. Le lieu de travail a été le mieux noté en France (76 \%). Le confort du travail a été loué de la même façon en France et au Portugal ( $81 \%$ ). Le salaire a obtenu la note la plus élevée en France (57 \%), ce score pouvant toutefois être qualifié de médiocre. Le score des avantages primes peut être qualifié de mauvais dans tous les pays et varie de 35 à $43 \%$. La relation personnelle avec l'employeur es la mieux perçue dans l'échantillon du Portugal $(77 \%$ ). La relation avec les collègues est évaluée de manière très égale dan tous les pays et varie de $79 \%$ à $82 \%$. La sécurité de l'emploi est étonnamment 
PROFIL DES ORGANISATIONS

D'ÉDUCATION POUR ADULTES DANS

LES PAYS RESPECTIFS

Image 9. Évaluation de l'organisation

Comparaison des notes selon le type d'organisations

$\begin{array}{lllllllllll}0 & 10 & 20 & 30 & 40 & 50 & 60 & 70 & 80 & 90 & 100\end{array}$

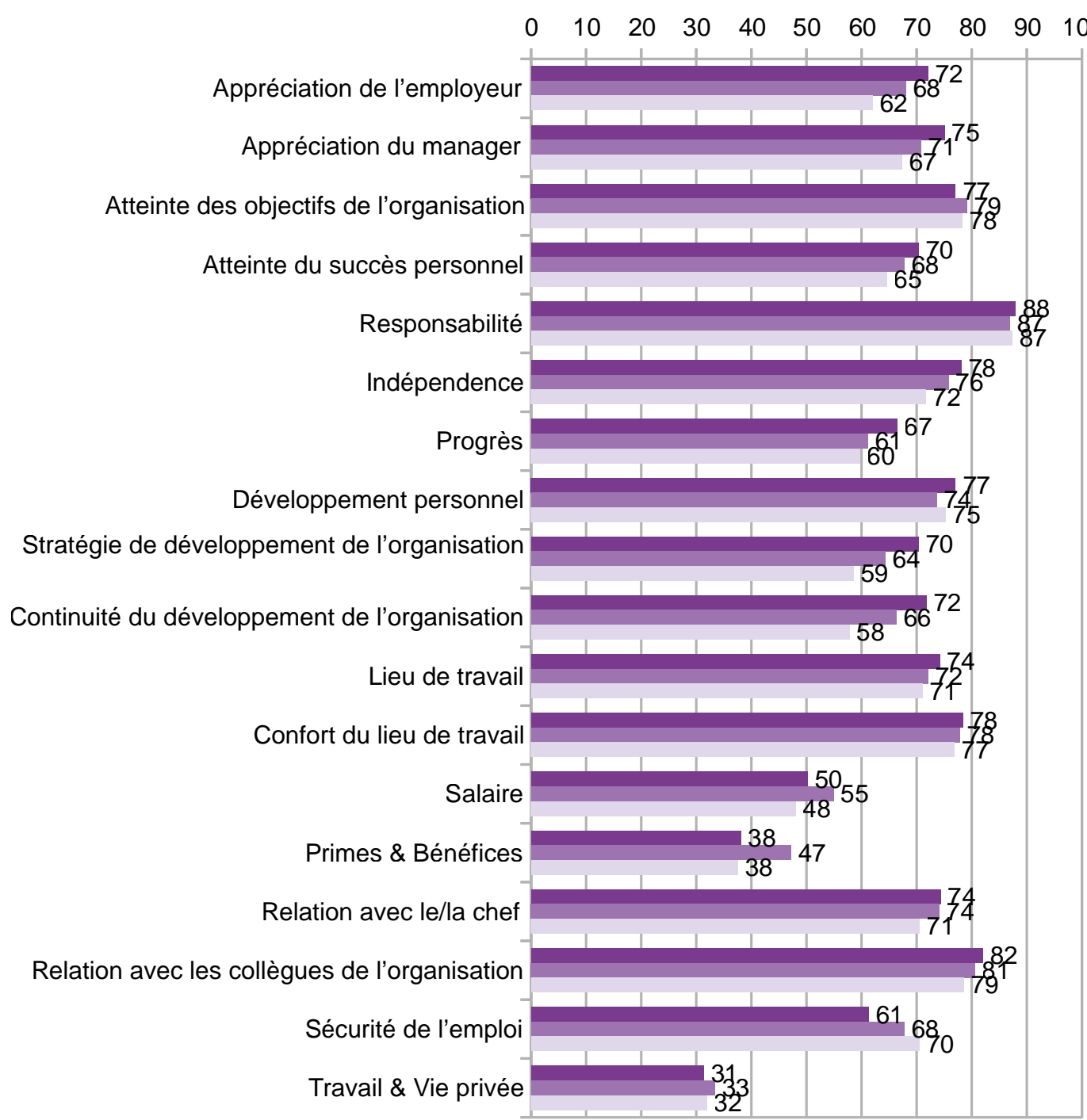

Sur la base des données collectées, il est possible de tirer des conclusions préliminaires concernant les types d'établissements d'enseignement pour adultes dans les pays partenaires du projet. Les personnes interrogées en Pologne ont également identifié différents types d'organisations (39 privées,

En France et en Italie, les organisations d'éducation pour adultes ont été principalement identifiées dans le secteur privé. En France, 32 publiques et $33 \mathrm{ONG}$ ) les répondants ont identifié 98 organisations privées, 39 publiques et 36 ONG, tandis qu'en Italie, il y avait 51 organisations privées, 44 publiques et 38 ONG.

Au Portugal, il y avait une disproportion entre le nombre d'organisations privées (54) et d'ONC (50), et les organisations publiques (34).
Cette comparaison indique que le secteur de l'éducation pour adultes des ONG était le moins représenté (le Portugal étant une exception), tandis que le secteur privé était le plus représenté. La plus grande disproporion portion entre les types dorganisations evaluées s'est produite en France, tandis que la plus petite en Pologne.

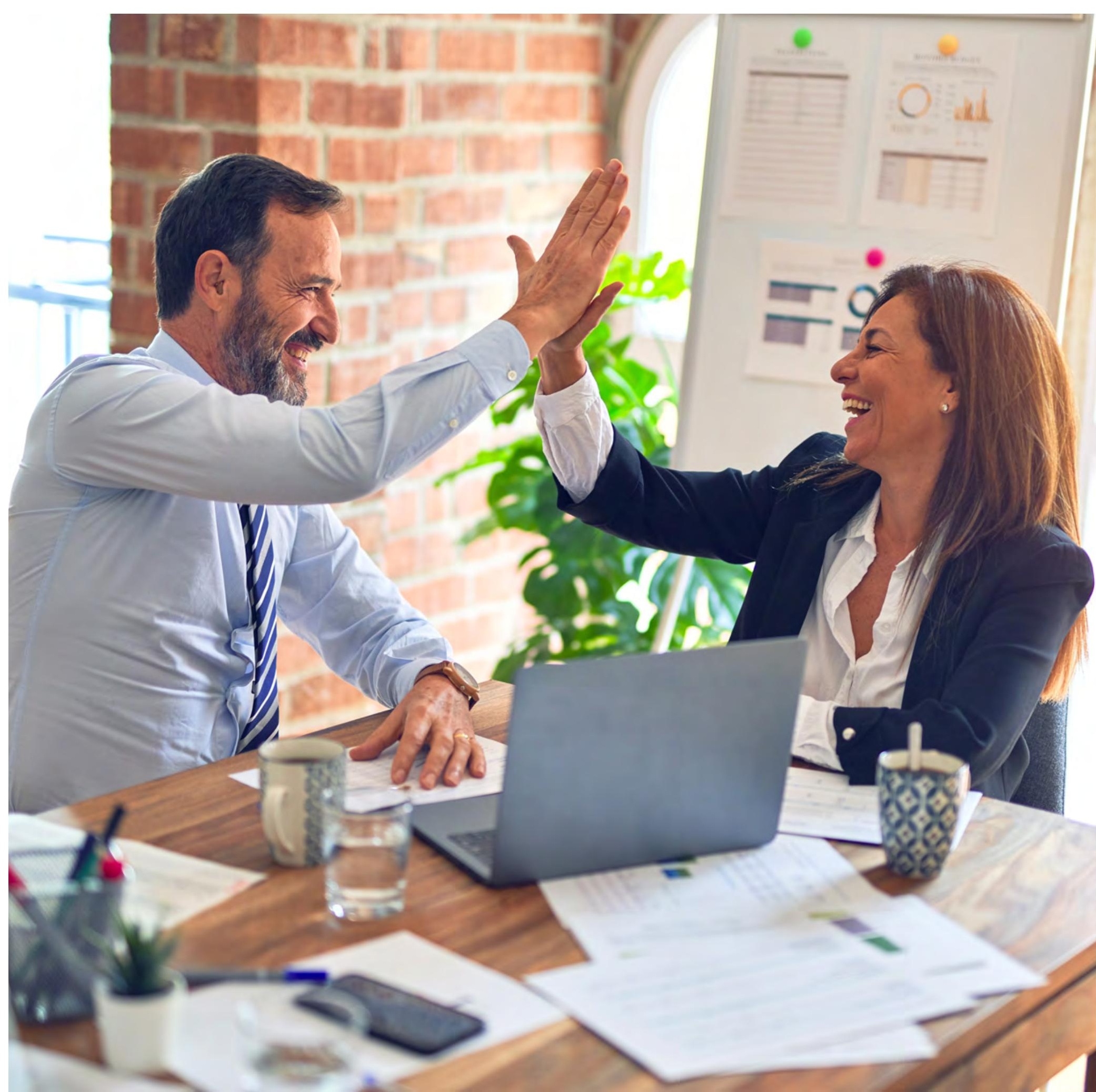


ÉVALUATION DES DIFFÉRENTS TYPES

D'ORGANISATIONS

Image 10. Type d'organisation évaluée par les répondants

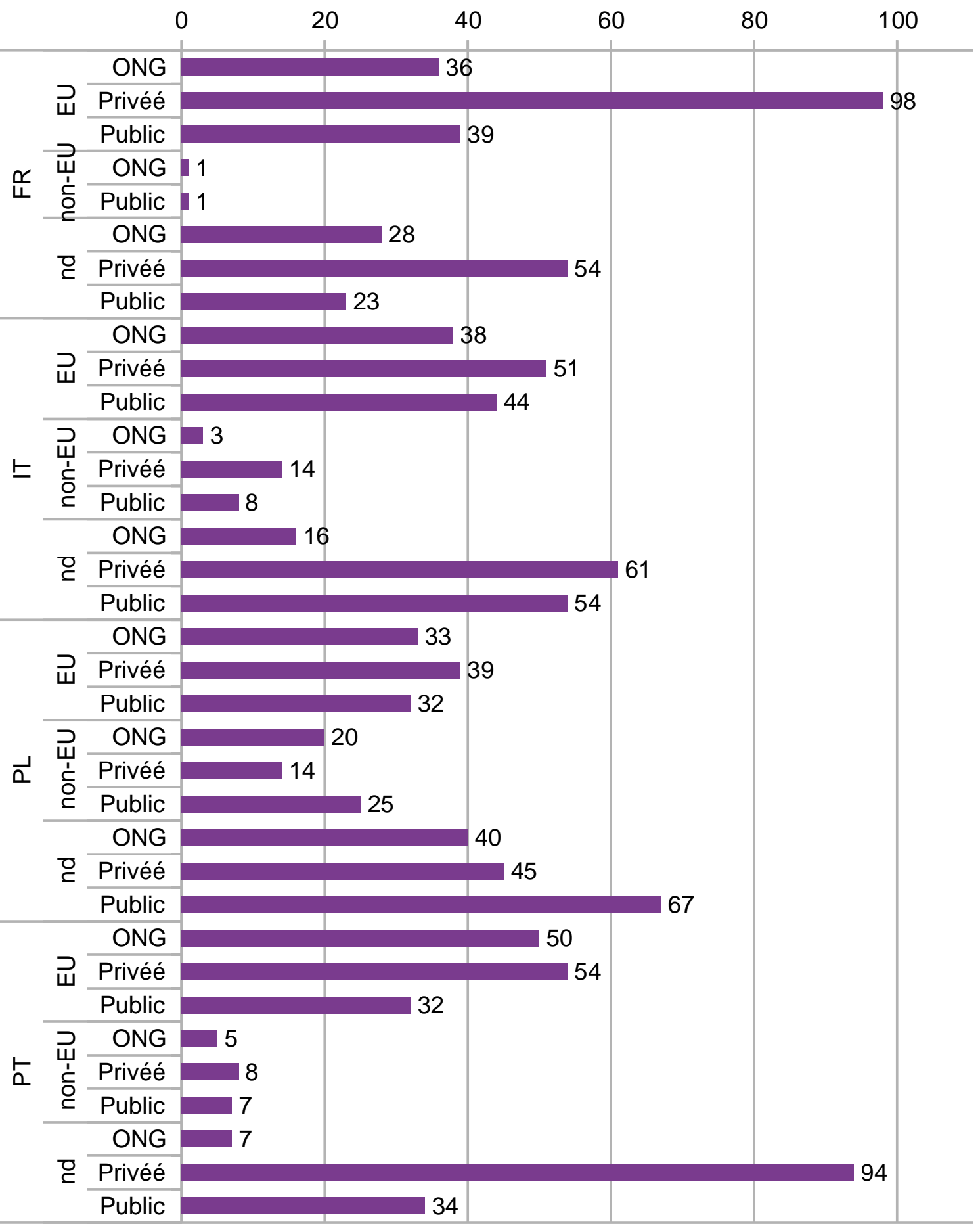

La comparaison de l'évaluation des différents types d'organisations concernan leur besoin de changement montre que, dans la plupart des cas, les organisations non gouvernementales sont mieux évaluées que les autres types d'organisations. Le besoin de changement le plus fort est indiqué par les répondants en ce qui concerne tutions publiques. Ces tendances diffèrent lorsqu'il s'agit d'indicateurs purement économiques. Les organisations non gouvernementales sont les moins bien notées en e qui concerne les salaires, les primes et la sécurité de l'emploi. Cela indique des besoins de changement plus importants à cet égard dans les ONG, par exemple sous la forme d'une aide financière ou d'une plus grande sécurité de l'emploi. 
3. Résumé

\section{RÉSUMÉ}


Notre projet de recherche visait à établir un diagnostic des besoins en matière d'éducation et à évaluer quantitativement les organisations. Il a intérrogé des éducateurs d'adultes et des travailleurs des ONG. Les résultats ont servi de base à trois analyses : 1) les scores généraux obtenus par les participants, 2) les scores généraux obtenus pa les citoyens de l'uE comparés à ceux obtenus par les citoyens des pays tiers et 3) les score généraux obtenus par les répondants des pays du consortium.

Au premier niveau - général - d'analyse, il a été révélé que les besoins éducatifs les plus faibles sont liés aux compétences numériques $(82 \%)$ et les plus élevés aux compétences entrepreneuriales (73\%). L'évaluation de l'organisation a montré que les participants étaient très satisfaits des responsabilités qu'ils avaient $(87 \%)$ et que la satisfaction la plus faible concernait les avantages et les primes ( $42 \%)$, et le salaire $(51 \%)$

Au deuxième niveau d'analyse - centré su la comparaison entre les citoyens de l'UE et les non citoyens de l'ue - il a été révélé qu le niveau de satisfaction des participants de l'ue était sensiblement plus élevé. Une exception a été notée dans les compétences multilingues et multiculturelles qui ont été évaluées plus favorablement par les citoyen non-UE.

L'évaluation de l'organisation indique une plus grande appréciation de l'organisation/ employeur par les citoyens de l'UE avec une exception notable dans la réussite perune exception notable dans la réussite per-
sonnelle $(+4 \%)$ et les avantages et primes $(+6 \%)$ en faveur des organisations non-UE.

Le troisième niveau d'analyse - centré sur les pays participants au consortium du projet a révélé que les besoins en matière d'éducation sont mieux satisfaits en France (71 \%).
Des scores plus faibles ont été notés en Italie et en Pologne (67\%). Les sous-catégorie ont révélé que : en France, les besoins les plus importants concernent les compétences entrepreneuriales ; en Italie et en Pologne, les besoins les plus importants concernen les compétences multilingues et multiculles compétences multilingues et multicul-
turelles et les compétences entrepreneuriales ; au Portugal, les besoins les plus importants concernent les compétences multilingues et multiculturelles, les compétences entrepreneuriales et l'ouverture à la science et à la culture.

En ce qui concerne l'évaluation de l'organisation, les scores varient tellement que seules les évaluations significatives ont été prises quence, les disproportions significatives ont été constatées dans les domaines suivants : 1) l'appréciation de l'employeur, 2) l'appréciation du supérieur hierarchique (chef/manager/superviseur) 3) la réussite personnelle, 4) la progression, 5) la stratégie de développement de l'organsation, 6) la co délo de lorganisalion, 7) les conditions de travail, 8) le confort du travail, 9) les conditions salariales, 10) les avantages et les primes, 11) les relations avec le chef, 12) la sécurite de l'emploi et 13) l'impact du travail sur la vie personnelle.

Les résultats montrent clairement que le niveau général des besoins deéducation des éducateurs d'adultes est satisfaisant; il en va de même pour l'évaluation des organisations. Cependant, il y a encore des possibilités d'amélioration et celles-ci se situent en général dans les domaines des compétences multilingues et multiculturelles et des compétences entrepreneuriales. Par conséquent, tous les futurs projets éducatifs visant à améliorer les besoins des éducateurs d'adultes devraient se concentrer sur ces domaines. Les principaux inconvénients des organisations employant des éduca- teurs d'adultes et des travailleurs du secteur de l'éducation des adultes se situent a niveau des salaires et des primes financière qui sont diagnostiqués à un niveau critique, sentiment de sécurité matérielle et qui peuvent être un facteur important pour attirer des talents dans ce secteur. En outre, l'attention es attirée sur le fait que le sous-financemen des institutions contrastent avec les compétences élevées des éducateurs.

Les besoins des citoyens des pays de l'uE ont généralement été satisfaits par rapport aux citoyens des pays tiers et ils fon

galement davantage l'éloge de leur organsation. Cependant, leur niveau de réussite personnelle est plus faible, ce qui implique que les éducateurs d'adultes et les travailleurs des ONG de l'UE ne lient pas aus (a) fortement len activice à leur réussite personnelle, ceci peut être expliqué par des facteurs culturels ou économiques.

Enfin, si l'on prend en compte uniquement les participants représentant les pays impliqués dans le projet, il existe une prémisse importante indiquant la nécessité de soutenir les changements dans les domaines suivants :

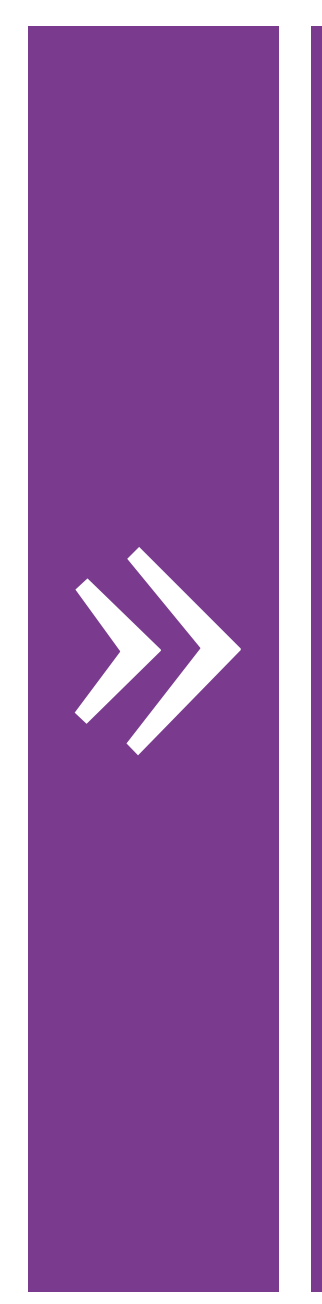

च appréciation de l’employeur, en particulier en ce qui concerne la Pologne

च appréciation du suppérieur hierarchique dans tous les pays à l'exception de la France ;

च la réussite personnelle, en particulier en ce qui concerne l'Italie ;

घ la progression, en particulier en Italie et en Pologne ;

『 la stratégie de développement de l'organisation, en particulier en Italie ;

च la continuité du développement de l'organisation, en particulier en Italie ;

च les conditions de travail et le confort, en particulier en Pologne ;

V les conditions salariales dans tous les pays diagnostiqués ;

च les avantages et les primes qui ont été mal évaluées ;

घ les relations personnelles avec le/la supérieur hiérarchique, en particulier en Italie ;

च la sécurité de l'emploi, en particulier en Pologne :

घ limpact du travail sur la vie privée, en particulier en Pologne. 
Le rapport a été évalué par une évaluatrice indépendante, Tijana Milenkovic Jankovic (UK). Elle a travaillé sur divers projets internationaux dans le domaine de l'éducation (continue et pour adultes) pendant plus de 20 ans. Cette expérience professionnelle lui a permis de mieux comprendre le fonctionnement des institutions et des organisations éducatives dans divers sec-

\section{Ses conclusions principales sont:}

1. L’AENI fournit un aperçu général solide des besoins des éducateurs d'adultes. Même si les résultats généraux du test AENI montrent principalement des résultats positifs dans tous les pays partenaires, les résultats son basés sur l'auto-évaluation. [...] Les résultats fournissent une indication aux décideurs politiques et parties prenantes sur les compétences les plus nécessaires pour les éducateurs d'adultes. Nous espérons qu'ils s'en servent dans le cadre de la planification de la formation initiale, ainsi que dans les cours de développement professionnel continu (DPC) pour les éducateurs d'adultes, mais aussi dans le cadre du processus de validassi dans le cadre du processus de validation des compétences acquises dans différents contextes (Résolution du Conseil, 2011) [...] Sur un plan général, la majorité des scores sontélevés. Toutefois, des besoins particulier teurs et à toutes les étapes du cycle éducatif. Elle a acquis une expérience dans le domaine de l'évaluation en tant que consultante indépendante dans le cadre de l'évaluation de projet du programme de formation professionnelle du HCR en Serbie et en tant que consultante régionale / nationale indépendante de Lux-Development au Monténégro.

sont identifiés concernant les compétences multilingues et multiculturelles (ML\&MC) et les compétences entrepreneuriales (CE) [...].

2. Il serait important que les organisations impliquées dans la recherche reçoivent les conclusions de l'étude et aient la possibilité comparer les résultats avec les résultats généraux au niveau national, mais aussi de les comparer avec d'autres pays. Cette recherche peut inspirer leur reflexion (basée sur leurs résultats) et les inspirer à établir des plans d'action pour améliorer la situation dans l'organisation [...].

3. L'instrument quantitatif développé pour l'évaluation des organisations a fourni une base valable pour la préparation de la recherche qualitative plus approfondie. 
4. References

\section{REFERENCES}

whe 
Bacigalupo, M., Kampylis, P., Punie, Y., \& Van den Brande, G. (2016). EntreComp: The entrepreneurship competence framework Luxembourg : Publication Office of the European Union, 10, 593884

Bassett-Jones, N., \& Lloyd, G. C. (2005),Does Herzberg's motivation theory have stayin power? Journal of Management Development, Vol. 24 Iss 10 pp. 929-943, http://dx doi.org/10.1108/02621710510627064

Buiskool, B. J., Broek, S. D., van Lakerveld, J.A Zarifis, G. K., \& Osborne, M. (2010). Ke competences for adult learning professionals. Contribution to the development of a reference framework of key competences for adult learning professionals, 157

Burns, D. (1985). Intimate connections. New York: Signet (Penguin Books)

Caena, F. (2013). Supporting teacher competence development for better learning outcomes. Education \& Training, European Commission, p. 5-59.

Council of Europe. Council for Cultura Co-operation. Education Committee. Modern Languages Division. (2001). Commo European Framework of Reference for Languages: learning, teaching, assessment. Cambridge University Press.

Council of the European Union. (2018). COUNCIL RECMMENDATIONS, 22 May 2018, with reference to key competences in the process of life-long learning. (2018/C 189/1). Officia Journal of the European Union, pp. 1-13

Council Resolution on a renewed European agenda for adult learning $\mathrm{OJ} C \mathrm{C} 32$, 20.12.2011, p. 1-6.

EU Science Hub - European Commission. 2020. Entrecomp: The Entrepreneurship
Competence Framework. - EU Science Hub - European Commission. [online] Available at: <https://ec.europa.eu/jrc/en/publication/eur-scientific-and-technical-researchreports/entrecomp-entrepreneurship-competence-framework $>$ [Accessed 15 November 2020].

Europa.eu. 2020. Common European Framework of Reference. Europass. [online] Available at: <https://europa eu/europass/en/common-european-frameworkreference> [Accessed 15 November 2020].

Faure, E., Herrera, F., Kaddoura, A. R., Lopez H., Petrovski, A. V., Rahnema, M., \& Ward, F. C. (1972). Learning to be: the world of education today and tomorrow. Paris: UNESCO.

Głomb, K. (2020). Edukacja dla Przemystu 4.o. Wyzwania dla Polski [Education for Indus-try 4.o A Challenge for Po land]. Warszawa: Agencja Rozwoju Przemystu.

Griffith, W. S. (1978). Educational Needs: Definition, Assessment, and Utilization. The School Review, 86(3), pp. 382-394.

Hakio, K., \& Mattelmäki, T. (2019). Future Skills of Design for Sustainability: An Awareness-Based Co-Creation Approach. Sustainability(11), pp. 1-24.

Herzberg, F., Mausner, B. and Snyderman, B. (1959), The Motivation to Work, Wiley, New York,NY.

Hipkins, R. (2018). How the key competencies were developed: The evidence base. Wellington: New Zealand Council for Educational Research.

Kobylarek, A. (2009). Kompetencje komunikacyjne w systemie umiejętności nauczytem of teachers' abilities]. In K. Błaszczyk,
M. Drzewowski, \& W. Maliszewski, Komunikacja spoteczna a zarządzanie we wspótczesnej szkole [Social communication and management in a modern school] (pp. 334342). Toruń: Adam Marszałek.

Martowska, K., \& Matczak, A. (2013). Pomiar kompetencji społecznych - prezentacj nowego narzędzia diagnostycznego [Measuring social skills - presentation of a new diagnostis tool]. Psychologia Jakości Życia [Psychology of the Quality of Life] (1) pp. 43-56.

Morris, E. (2001). Special Educational Needs. Code of Practice. London: Department for Education and Skills.

Nijssen, A., van Lakerveld, J. A., Buiskool, B. J, den Oudendammer, F., Broek, S. D., \& Hake, B. (2008). ALPINE - Adult Learning Profession in Europe: A Study of the Current Situation, Trends and Issues. Project Report. Research voor Beleid, Zoetermeer, Netherlands.

Padzik, K. (2016). Ocena zintegrowana Assessment i Development Center. Warszawa: Wolters Kluwer.

Paterson, R. (2000). The Assertiveness Workbook: How to Express Your Ideas and tionships. Oakland: New Harbinger.

Petrètiène, A., Daukšienė, J., \& Grašienė, J. (2020). Creativity and speciality language in the context of the development of key competences. Mokslas - Lietuvos ateitis(12), pp. 1-7.

Radovan, M. (2019). Cognitive and Metacognitive Aspects of Key Competency "Learning to Learn”. Pedagogika(1), pp. 28-41.

Stufflebeam, D. L., McCormick, C. H., Brinkerhoff, R. O., \& Nelson, C. O. (2012). Conducting Educational Needs Assessments. Springer.
Warzocha, T. (2016). Kompetencje komunikacyjne jako komponent kompetencji społecznych nauczycieli akademickich - założenia do badań [Communication skills as a component of social skills for academic teachers - a foudation for research]. Edukacja Technika - Informatyka [Education - Engineering - Information Technologies] (2), pp. 70-75 
5. Annexes

\section{ANNEXES}

whe 
(Adults Education Needs Inventory - Inventaire des besoins des éducateurs d'adultes) Questionnaire pour étudier les besoins de formation dans le domaine des compétences clés des adultes

Vous trouverez ci-dessous une liste de 39 affirmations. Veuillez indiquer dans quelle mesure vous êtes d'accord avec chaque affirmation sur une échelle de 1 à $5(1=$ absolument pas d'accord, $5=$ Il n'y a pas de bonnes ou de mauvaises répon-

ses. Ne passez pas trop de temps sur chaque affirmation, mais donnez la réponse qui vous vient à l'esprit en premier. Cette recherseront utilisés exclusivement entifiques.

\section{NOTE : Répondez à toutes les affirmations}

= absolument pas d'accord, 5 = tout à fait d'accord

CC Je peux instaurer un dialogue dans n'importe quelle 1 situation (par exemple, lors d'un conflit ou lorsque mon interlocuteur a un avis différent)

CC Je considère que je peux transmettre des messages clairs et compréhensibles

SCC Lorsque je cherche des informations, j'essaie d'utiliser différentes sources

CC Je suis très souvent confronté(e) à des situations où il est

difficile d'exprimer mes pensées

5 CC Je n’ai généralement pas de problème pour comprendre mon interlocuteur

6 CC J'aime discuter avec différentes personnes

7 CC Je me sens à l'aise quand je parle avec d'autres personnes

CC Je peux exprimer mes pensées d'une manière non

conventionnelle

MM Je parle et écris couramment plus d'une langue étrangère

$10 \mathrm{R}$ OSC Je considère que je n’ai pas besoin de développer ma

connaissance des langues étrangères

11 MM Je saisis différentes opportunités pour apprendre des langues étrangères

12 MM J'aime apprendre de nouvelles langues 
$1=$ absolument pas d'accord, 5 = tout à fait d'accord

13. MM J'utilise une langue étrangère tous les jours (par

exemple, en regardant des films, en lisant des livres)

14R OSC Je considère que les mathématiques n’aident pas

à découvrir la vérité

OSC Il existe des doutes justifiés concernant certaines théo-

$15 \mathrm{R}$ ries, par exemple l'influence humaine sur le climat ou l'efficacité des vaccins

16 EC Je considère qu'une action efficace nécessite un plan clair et sans ambiguitté

17R EC J'agis parfois de manière illogique

18 SCC J'utilise souvent diverses ressources lors de la planifica-

tion d'une action

19 EC Je suis généralement un plan préétabli

DC Je sais comment utiliser les nouvelles technologies pour

une communication plus efficace

21 DC J'utilise diverses innovations technologiques

22 DC Je pense que je suis suffisamment compétent en matière

de technologies de l'information

DC Je peux facilement utiliser les appareils les plus courants

(PC, smartphone, ordinateur portable, tablette, etc.)

$24 \quad \mathrm{CC}$ Je peux travailler en groupe

CC Je pense qu'il est difficile pour moi de nouer des relations

avec d'autres personnes

26R EC Je suis parfois compliqué(e) dans mes relations avec les

OSC Il est impossible de concilier la politique d'intégration

de l'UE avec les spécificités culturelles

28 SCC Je m’engage dans des questions sociales qui sont impor-

tantes pour moi

29 SCC Je sens que jai un effet sur mon environnement

CC Je peux motiver les autres à réaliser des objectifs communs

$31 \quad$ CC Je suis efficace dans les négociations

OSC Le monde n’offre pas beaucoup de possibilités pour

réaliser mes idées
$1=$ absolument pas d'accord, 5 = tout à fait d'accord

33R EC Je n'aime pas la phase d'organisation et planification

EC Je considère que le travail de contrôle et de surveillance est généralement inutile

35R EC J'ai parfois le sentiment de mal gérer mes tâches

36R CC Je fais rarement des retours à mes collègues

$37 \quad$ MM Je m’intéresse aux différentes cultures

38R OSC Je ne serais pas en mesure de m'exprimer artisti-

quement

39R OSC Pour moi, la culture contemporaine ne vaut rien

CC Compétences en matière de communication (max. 60 pts.): 1, 2 $4 \mathrm{R}, 5,6,7,8,24,25 \mathrm{R}, 30,31,36 \mathrm{R}$

MM Multilinguisme et multiculturalisme (max. 25 pts.): 9, 11, 12, 13, 37

DC Compétences numériques (max. 20 pts.): 20, 21, 22, 23

EC Compétences en matière d'entrepreneuriat (max. 35 pts.): 16, 17R, 19, 26R, 33R, 34R, 35R

OSC Ouverture à la science et à la culture (max. 35 pts.): 10R, 14R, 15R, 27R, 32R, 38R, 39R

SCC Compétences sociales et civiles (max. 20 pts.): 3, 18, 28, 29

R Score inversé
INTERPRÉTATION DES RÉSULTATS

Une recherche normalisée est actuellement menée sur un groupe représentant les éducateurs de divers pays européens. À ce stade, les auteurs proposent l'utilisation de normes provisoires pour aider à la conversion des En fonction du nombre de points gagnés dans chaque catégorie, il est possible d'attribuer les résultats à l'un des trois groupes - élevé, moyen et faible. Des résultats faibles indiquent un faible niveau de compétence chez un éducateur particulier, et donc un besoin accru d'améliorer cette compétence.

Interprétation des résultats

\begin{tabular}{|lccc|}
\hline & faible & moyen & élevé \\
\hline CC & $12-13$ & $32-41$ & $42-60$ \\
\hline MM & $5-13$ & $14-17$ & $18-25$ \\
\hline DC & $4-10$ & $11-14$ & $15-20$ \\
\hline EC & $7-18$ & $19-24$ & $25-35$ \\
\hline OSC & $7-18$ & $19-24$ & $25-35$ \\
\hline SCC & $4-10$ & $11-14$ & $15-20$ \\
\hline
\end{tabular}




\section{EVALUATION DE L'ORGANISATION}

Questionnaire examinant la nécessité de changements dans les activités de l'organisation

Vous trouverez ci-dessous 18 affirmation concernant votre opinion sur votre lieu de travail. Veuillez indiquer dans quelle mesure

vous êtes d'accord avec chaque affirmation sur une échelle de $\mathbf{1}$ à 5 ( 1 = absolument pas d'accord, 5 = tout à fait d'accord)

\section{NOTE : Veuillez répondre à CHAQUE question}

1 = absolument pas d'accord, 5 = tout à fait d'accord $\begin{array}{lllll}1 & 2 & 3 & 4 & 5\end{array}$

\section{Annex 11}


1 = absolument pas d'accord, 5 = tout à fait d'accord $\begin{array}{lllll}1 & 2 & 3 & 4 & 5\end{array}$

54 Ma relation directe avec mon hiérarchie est satisfaisante

55 Mes relations directes avec mes collègues sont satisfaisantes

56 Mon organisation me garantit la sécurité de l'emploi

57 Mon travail a un effet négatif sur ma vie privée

Veuillez indiquer

58. Sexe $\square \mathrm{M} \quad \square \mathrm{F}$

59. Age ...........

60. Type d'organisation:

$\square$ Public

$\square$ Privéé

$\square$ ONG

61. Poste occupé (veuillez n'en marquer qu'un seul par un X)

\begin{tabular}{l|l}
\hline $\begin{array}{l}\text { Fondateur (ice) / directeur } \\
\text { (ice) de l'organisation }\end{array}$ & \\
\hline Cadre intermédiaire & \\
\hline Employé(e) & \\
\hline Bénévole & \\
\hline
\end{tabular}

INTERPRÉTATION DES RÉSULTATS

Les scores sont calculés en pourcentages \%. Chaque élément peut avoir une note minimale de o $\%$ et une note maximale de 100 $\%$. Plus le score est élevé, plus l'opinion de 'individu sur son organisation est positive. La seule exception est la dernière catégorie a suterie axée sur le tranil et la vie privé. Dans cette catégorie, plus le score obtenu est bas, plus linfluence du travail sur la vie privée est faible, donc plus le résultat est positif.

Il est recommandé d'interpréter les scores obtenus en matiere dévaluation de lorganisation selon la classification proposée ci-dessous :

$\mathbf{0 - 2 0} \%$ - très faible - très mauvaise opinion de l'organisation/employeur

21-40\% - faible - mauvaise opinion de l'organisation/employeur

41-60\% - moyen - opinion neutre sur l'organisation/l'employeur

61-80\% - élevé - bonne opinion de l'organisation/employeur

81-100\% - très élevé - très bonne opinion sur l'organisation/employeur

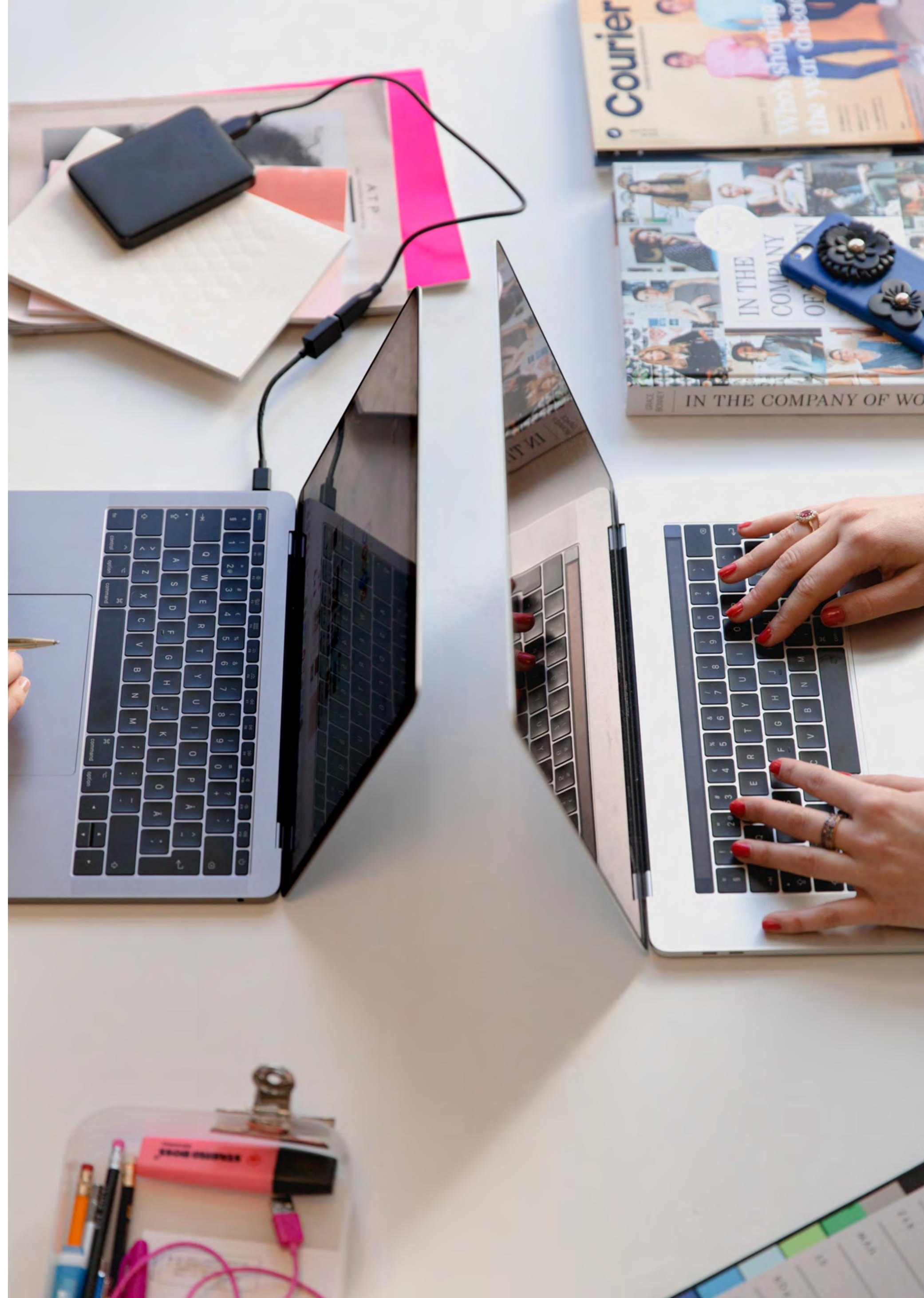



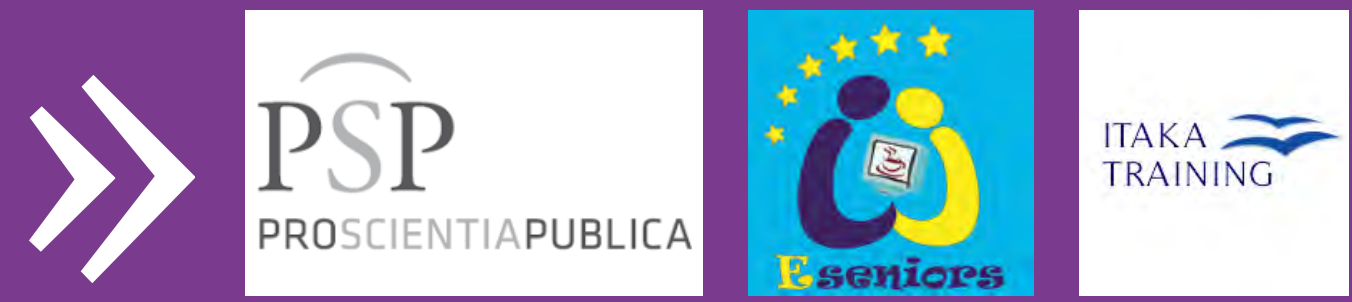

ISBN 978-83-953451-3-5

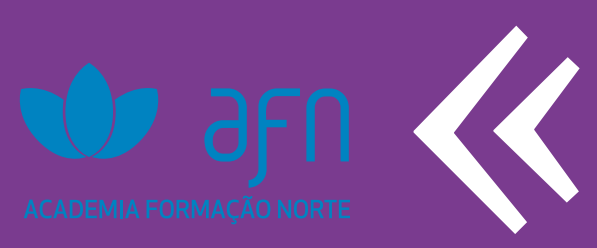

